\title{
Targeted Drug Delivery - From Magic Bullet to Nanomedicine: Principles, Challenges, and Future Perspectives
}

\author{
Ashagrachew Tewabe $\mathbb{D}^{\prime}$ \\ Atlaw Abate (iD) ${ }^{2}$ \\ Manaye Tamrie $\mathbb{1}^{3}$ \\ Abyou Seyfu (D) $^{3}$ \\ Ebrahim Abdela Siraj (D) \\ 'Department of Pharmacy, College of \\ Medicine and Health Sciences, Bahir Dar \\ University, Bahir Dar, Ethiopia; ${ }^{2}$ Ethiopian \\ Food and Drug Authority (EFDA), \\ Federal Ministry of Health (FMoH), Addis \\ Ababa, Ethiopia; ${ }^{3}$ Department of \\ Pharmaceutics and Social Pharmacy, \\ School of Pharmacy, College of Health \\ Sciences, Mizan-Tepi University, Mizan- \\ Aman, Ethiopia
}

\begin{abstract}
Nanomedicine is an advanced version of Paul Ehrlich's "magic bullet" concept. Targeted drug delivery is a system of specifying the drug moiety directly into its targeted body area (organ, cellular, and subcellular level of specific tissue) to overcome the aspecific toxic effect of conventional drug delivery, thereby reducing the amount of drug required for therapeutic efficacy. To achieve this objective, the magic bullet concept was developed and pushed scientists to investigate for more than a century, leading to the envisioning of different nanometer-sized devices - today's nanomedicine. Different carrier systems are being used and investigated, which include colloidal (vesicular and multiparticulate) carriers, polymers, and cellular/subcellular systems. This review addresses the need for and advantages of targeting, with its basic principles, strategies, and carrier systems. Recent advances, challenges, and future perspectives are also highlighted.
\end{abstract}

Keywords: targeting, polymers, nanoparticles, carriers, nanosomes

\section{Introduction}

Drug delivery (DD) refers to the methods, formulations, technologies, and processes involved in transporting a pharmaceutical substance in the body to achieve the desired therapeutic effect. ${ }^{1}$ It encompasses the approaches of administering medicinal compounds in humans and animals to attain therapeutic effectiveness. Recent developments in drug delivery systems (DDSs) are primarily been focused on smart DD, which focuses on drug administration at the appropriate time, dosage, and location with maximum safety and efficacy. ${ }^{2}$ The advancement of novel DDSs (NDDSs) has attracted pronounced attention in recent years. These systems enhance the therapeutic effectiveness of new and existing drugs with targeted, managed, and sustained delivery while meeting real and appropriate drug demand. ${ }^{1}$ DD is a growing field in pharmaceutical science. There are five generations of DDSs, and targeted delivery belongs to the fourth generation. ${ }^{3}$ Figure 1 illustrates the generations of DDSs. Over the last few decades, developing sustained or controlled DDSs has been a focus, with the objective of controlling and/or sustaining drug release, reducing dose frequency, or increasing drug efficacy compared to conventional delivery. Bilayer tablets are one example of an NDDS, used with modification of conventional drug-preparation and -delivery approaches. They are composed of two of the same drug or different drugs fixed in a single dose for sequential release of the combined drugs or sustained and
Correspondence: Ashagrachew Tewabe Department of Pharmacy, College of Medicine and Health Sciences, Bahir Dar University, PO Box 79, Bahir Dar, Ethiopia Tel +251 9l-296-0525

Email ashutewabe@gmail.com 


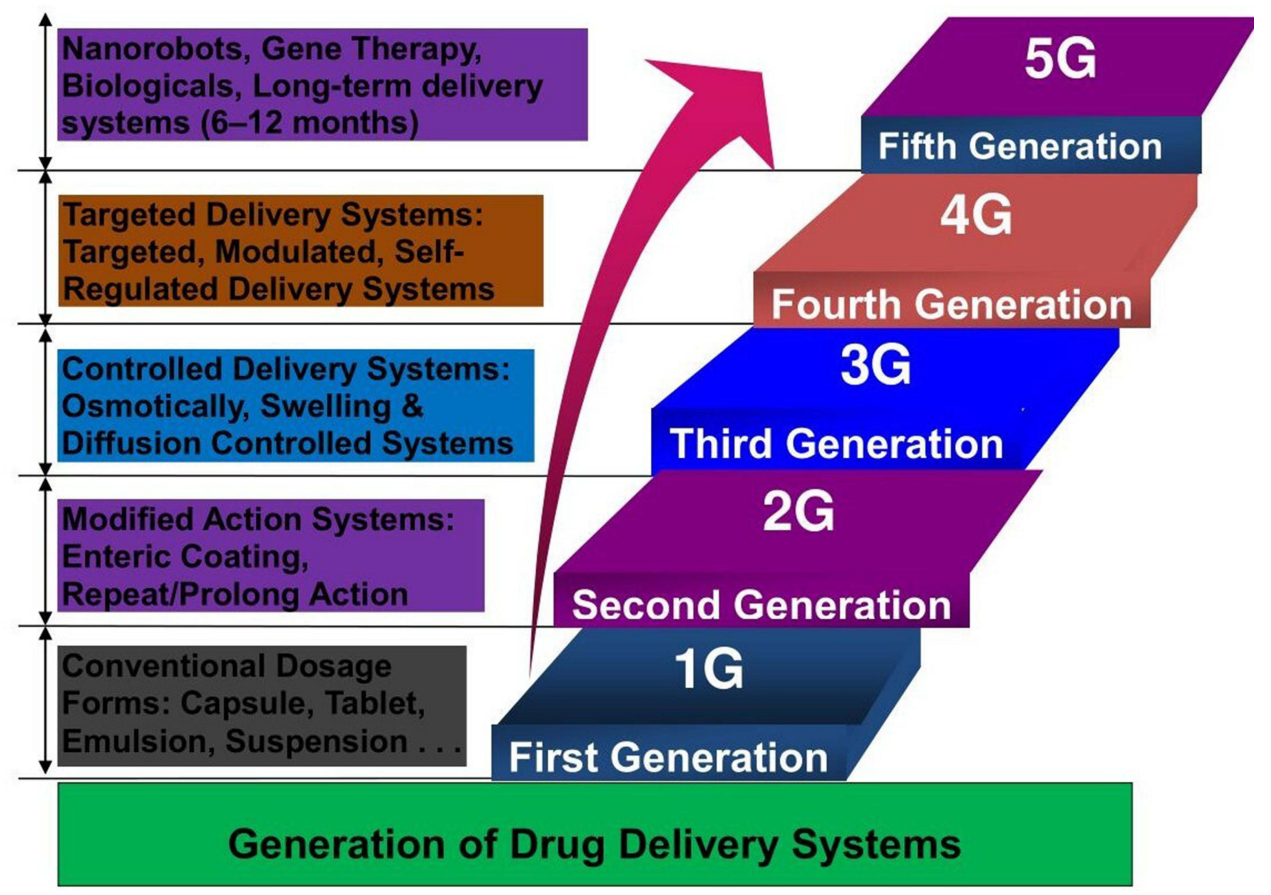

Figure I Generation of drug-delivery systems.

immediate release of the same drug, one as a loading dose and other as a maintenance dose. ${ }^{4}$ Such modifications in all forms of traditional DD can represent promising advancements, but there are still types of DDSs that need to be refined, such as delivery of poorly soluble drug formulations, protein delivery, self-regulated insulin delivery, and targeted DDSs (TDDSs). Targeted delivery to tumors is another potential advance that can be achieved with nanotechnology-based DSs. ${ }^{5}$ Nanoparticle (NP)-based DD brings an opportunity for controlled release of drugs, allowing sufficient time for drugs to act with enhanced therapeutic action and respond to specific stimuli, such as $\mathrm{pH}$, light, heat, or enzymes. ${ }^{6}$

TDDSs are where a drug is delivered to a specific location, rather than the whole body or organ, and combine diverse fields of science, such as polymer science, pharmacology, bioconjugate chemistry, and molecular biology. TDD is aimed at managing and controlling the pharmacokinetics, pharmacodynamics, aspecific toxicity, immunogenicity, and biorecognition of therapeutic agents. ${ }^{7}$ The end goal is improving treatment effectiveness while reducing side effects. TDDSs differ from conventional or traditional DDSs in that they acquire site-specific release of drugs from a dosage form, while the former depends on drug absorption through biological membranes. ${ }^{8}$

\section{The Concept of the Magic Bullet}

The concept of targeting of drugs to their site of action dates back to the postulation of the "magic bullet" concept." A century ago, Paul Ehrlich envisioned the concept of selectively targeting a pathogen without harming the host organism using "magic bullets." Analysts in cancer treatment were particularly inspired by the idea. ${ }^{10}$ Ehrlich approached his magic bullet concept in two consecutive steps: screening for toxic drugs, followed by modifying toxic drugs to be more specific and less toxic. ${ }^{11}$ He strongly pictured that achieving a cure would be very stress-free with substances that had exclusive affinity toward the causative bacteria alone, with no affinity for the host. This would finally result in the least harmful effect on the human body by exerting an exclusive lethal action on the parasite within the organism, hence the term "magic bullet."12 Ehrlich anticipated site-specific therapies to acquiring knowledge on how to cast magic bullets, as the magic bullets of a gunman hit the enemies exclusively. This fascinating idea pressed scientists to investigate further for more than a century, and led to the discovery of different nanometerscale devices, called "nanomedicines" nowadays. ${ }^{13}$ The success of this concept is a good indicator of its appeal, but implementing the magic bullet in the clinic remains a challenge. This is due to difficulties in finding the right 
target for a specific disease state, the medication that efficiently cures the disease, and the way to deliver the drug in a stable form to specific sites while preventing immunogenic and aspecific interactions. NPs are potentially useful as carriers of active drugs, and when coupled with targeting ligands, may fulfill many attributes of a magic bullet. ${ }^{9}$ According to the magic bullet philosophy of Ehrlich, drugs should go straight to their anticipated targets in the body and only interact with the target molecule. However, drugs pass complex pathways and contacts during their transport to reach their targets and possibly interactwith multiple targets, resulting in side effects. Unfortunately, there has never been a drug or aDDS that has directly reached the bodily target without these pathway interactions. This interference with several targets makes the drug a "magic shotgun," rather than a magic bullet. To meet the magic bullet target, we still have a long way to go. ${ }^{14}$

\section{The Need for Targeted Drug Delivery}

The need for TDD over conventional DSs is fourfold: unsatisfied peformance of drugs in terms of pharmacodynamic, pharmacokinetic, pharmaceutical, and pharmacotherapeutic features with conventional delivery, as shown in Figure 2. Targeting of drugs to a particular area through optimized DD methods is not only important to enhance therapeutic effectiveness but also to reduce the toxicity associated with a small therapeutic index and high doses. ${ }^{15}$ Targeting is needed to achieve solutions to these constraints and innate disadvantages of conventionalDDSs. Parenteral delivery is highly invasive, oral administration cannot be used for protein- or peptidederived drugs, and topical creams and ointments are limited to local effects. Furthermore, the effectiveness of drug-target interactions is compromised unless the drug is delivered to its site of action at a dosage and rate that produces minimal side effects while maximizing therapeutic effects. ${ }^{8}$ In addition, simpler drug-administration procedures, decreased drug quantity, which reduces therapeutic costs, and the potential to sharply increase drug concentration in target compartments without adverse effects on nontarget compartments are promising benefits of TDD. Generally, drug targeting results in increased efficacy, modulated pharmacokinetics, controlled biodistribution, increased specificity of localization, decreased toxicity, reduced dose, and improved patient compliance. . $^{8,16}$

\section{Basic Principles and Applications of Targeted Drug-Delivery Systems}

The basic principle behind drug targeting is delivering a high concentration of drug to the targeted site while minimizing its

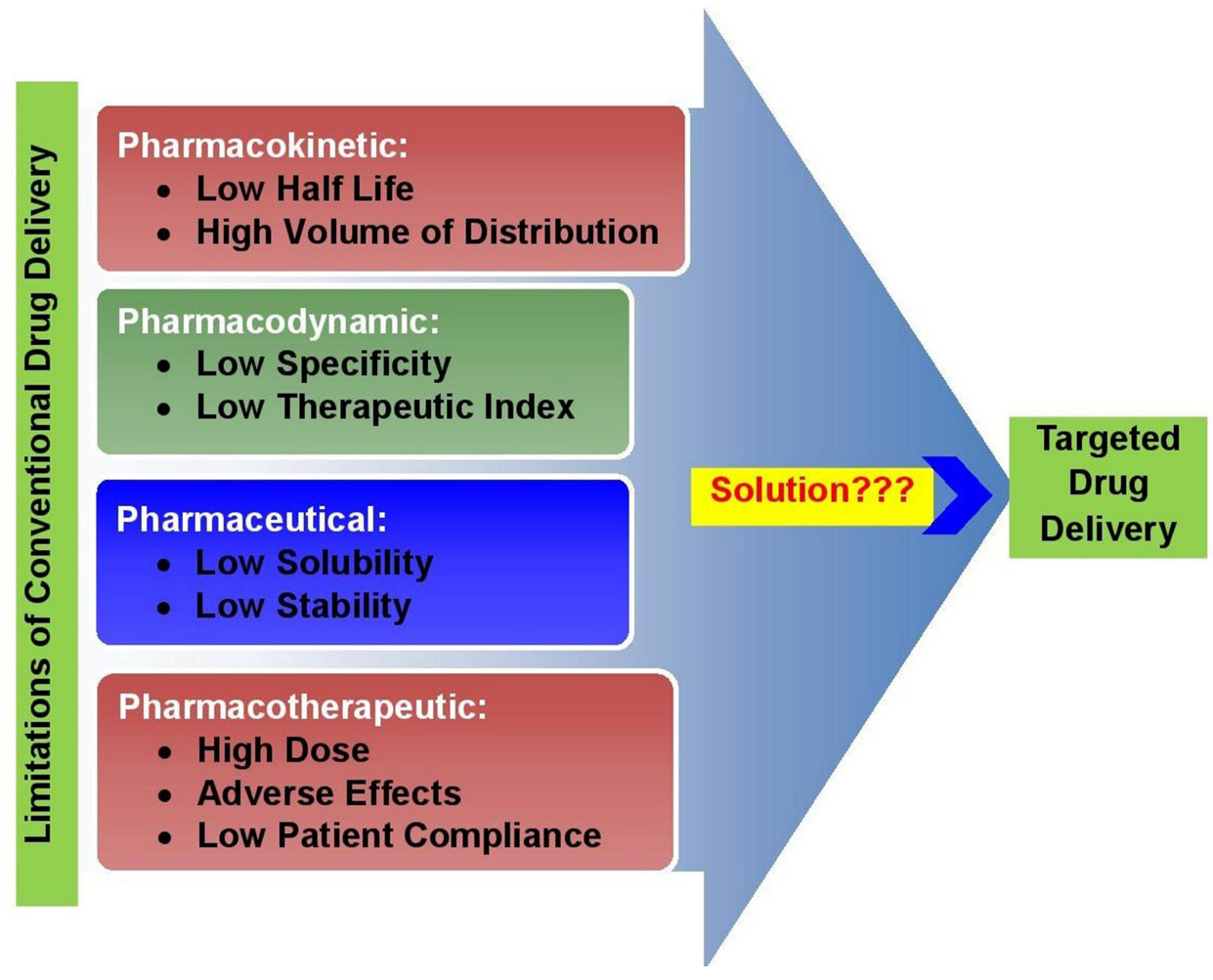

Figure 2 The need for targeted drug delivery. 
concentration to the nontargeted region. This principle aids in optimizing the drug's therapeutic effects while decreasing the side effects due to multitarget interactions, higher doses, and nontarget concentrations. ${ }^{17}$ Targeting also ameliorates unwanted interactions of the drug with bioenvironmental factors that affect drug access to targeted sites in the body, as shown in Figure 3. ${ }^{18}$ Drug targeting comprises coordinated drug behavior, targeting site, and pharmaceutical carrier. The target is the specific organ, a cell, or group of cells in chronic or acute condition demanding treatment with which the drug is going to interact. The carrier is a specially engineered molecule or system essential for effective transportation of the loaded drug toward preselected sites. ${ }^{19}$ Ideally, a drug-targeting complex is expected to be atoxic, nonimmunogenic, biochemically inert, biodegradable, biocompatible, and physicochemically stable in vivo and in vitro. It should also have a predictable and controllable pattern of drug release, reasonably simple, reproducible, and cost-effective preparation, be easily and readily eliminated from the body, and minimal drug leakage during transit. ${ }^{16,20}$

In order to assure the fulfillment of these ideal characteristics, targeted drug products should be prepared while considering the specific properties of target cells and the nature of transport carriers or vehicles that convey the drug to specific receptors. These considerable parameters include drug concentration, particulate location and distribution, molecular weight, physicochemical properties, enzymes, electric fields, physiological environment, nature/concentration of polymers/excipients, and surface morphology (shape, charge, size, and density) of the

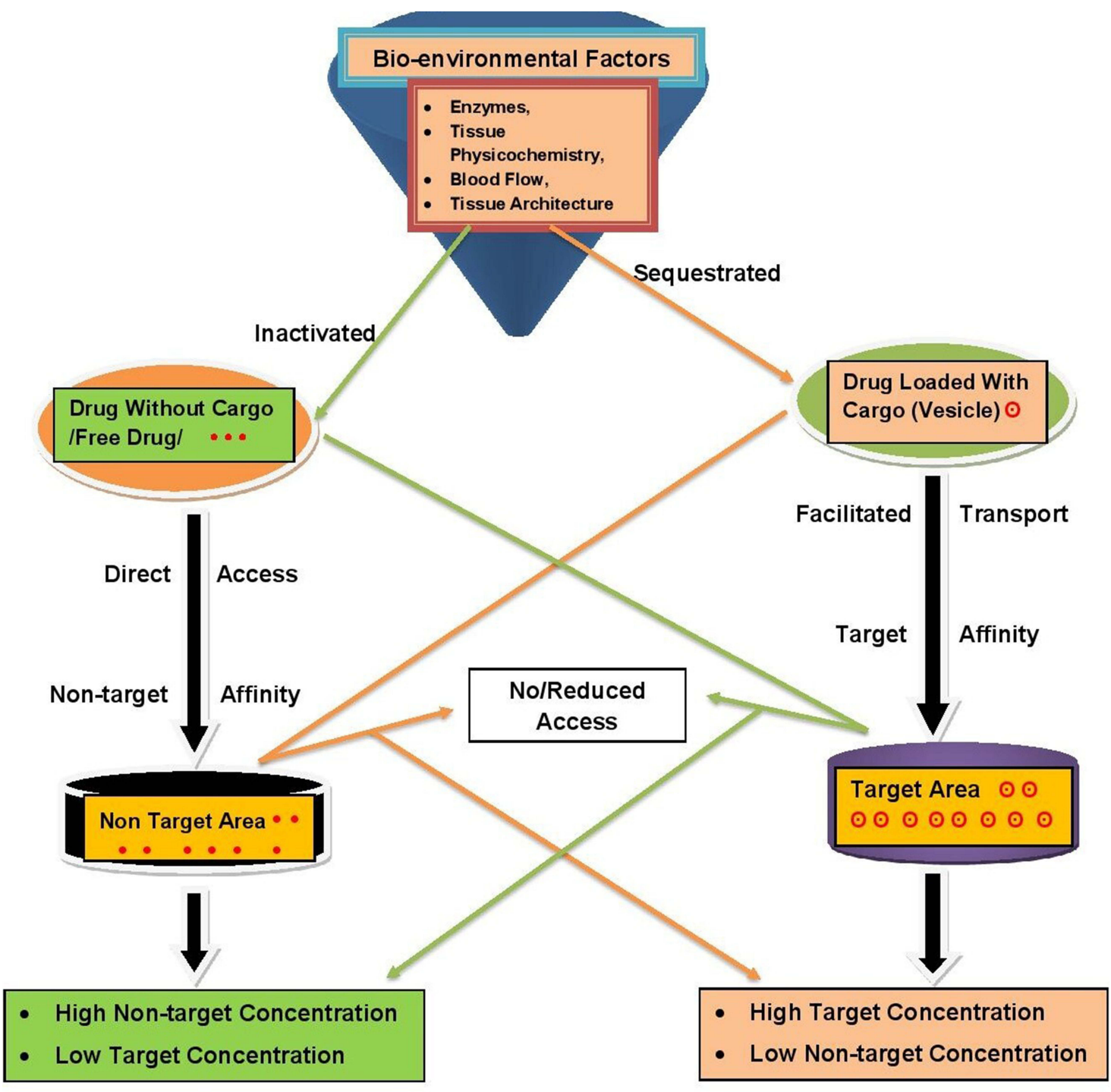

Figure 3 Principles of drug targeting. 
carrier system. ${ }^{16}$ Physiological variables, such as blood flow for intravenous administration of drugs and tissue architecture, along with physicochemical parameters, including carrier geometry, avidity, composition, and functionalization, should be controlled for effective targeting of desired cells or tissue. ${ }^{21}$ In addition, the clinical enhanced permeability and retention (EPR) effect, extravasation, intratumoral distribution, tumor heterogeneity, and overexpression characteristics are important factors in effective tumor-targeted treatment. ${ }^{14}$ If the ideal properties are well met and formulation factors well considered, TDD can be applied effectively in innovative nanomedicine and therapeutics. Though TDD can be used to treat many chronic and infectious diseases, its most important application is in treating cancerous tumors, due to its better microphage penetration and enhanced concentration at the infection site. ${ }^{3}$ Promising applications and purposes of TDD include cancer therapy, vaccine adjuvant, ocular and brain delivery, DNA and oligonucleotide delivery, intracellular and systemic targeting, oral and transdermal delivery, enzyme immunoassays, and radioimaging. Generally, outcomes reported with these applications include reduced toxicity, enhanced uptake, prolonged systemic circulation with enhanced bioavailability and drug effect, enhanced immunoresponse, improved drug absorption and permeation, and improved drug retention or reduced washout. ${ }^{20}$

\section{Types of Targeted Drug-Delivery Systems}

Various approaches are used to help target specific body sites, as depicted in Figure 4.

\section{Active and Passive Targeting}

Passive targeting is DD that targets systemic circulation. In this technique, drug targeting occurs because of the body's natural response to physicochemical characteristics of the drug or drug-carrier system. This is based on the accumulation of $\operatorname{drug}(s)$ at areas targeting the site of interest, such as in the case of tumor tissue. ${ }^{3}$ NPs are used as carriers in passive targeting, and they are directed to enter blood vessels more at the disease site, which provides the opportunity for significant drug accumulation at the target. This process is aided by slow lymphatic drainage - the EPR effect. ${ }^{6}$ On the other hand, active targeting is a particular ligand receptor-type interaction that occurs after blood circulation and extravasation. ${ }^{15}$ It mainly relies on the biological interface between target cells and the ligands attached to NPs. Various types of ligands have been employed for this purpose, including proteins, polysaccharides, nucleic acids, peptides, and small molecules. ${ }^{6,8}$ Figure 5 illustrates the differences between the two targeting approaches.

Tumors are characterized by highly defective vessel architecture and poor lymphatic drainage, in accordance with the EPR effect. Small nanocarriers with those tumor

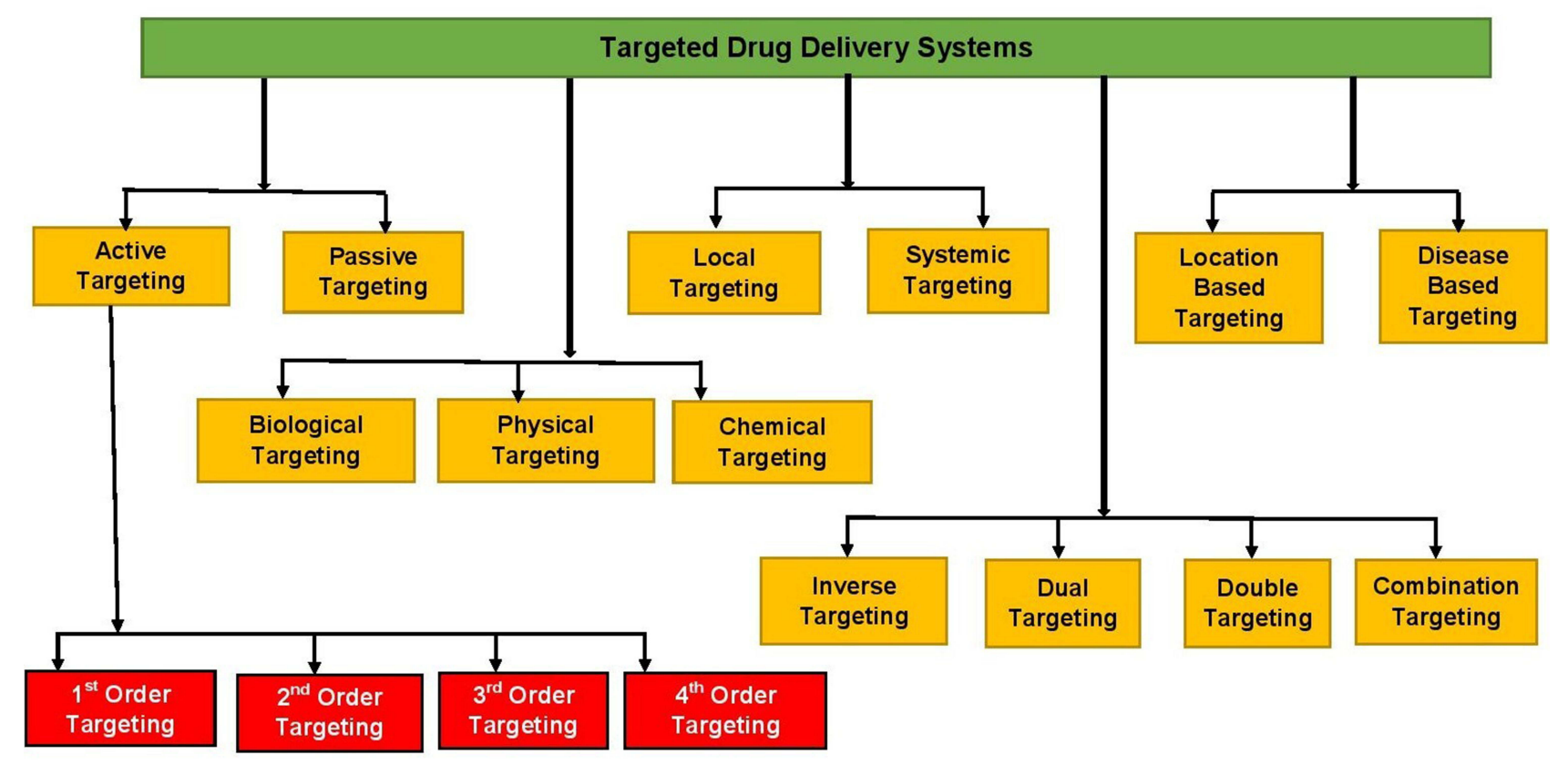

Figure 4 Various categories of drug targeting. 


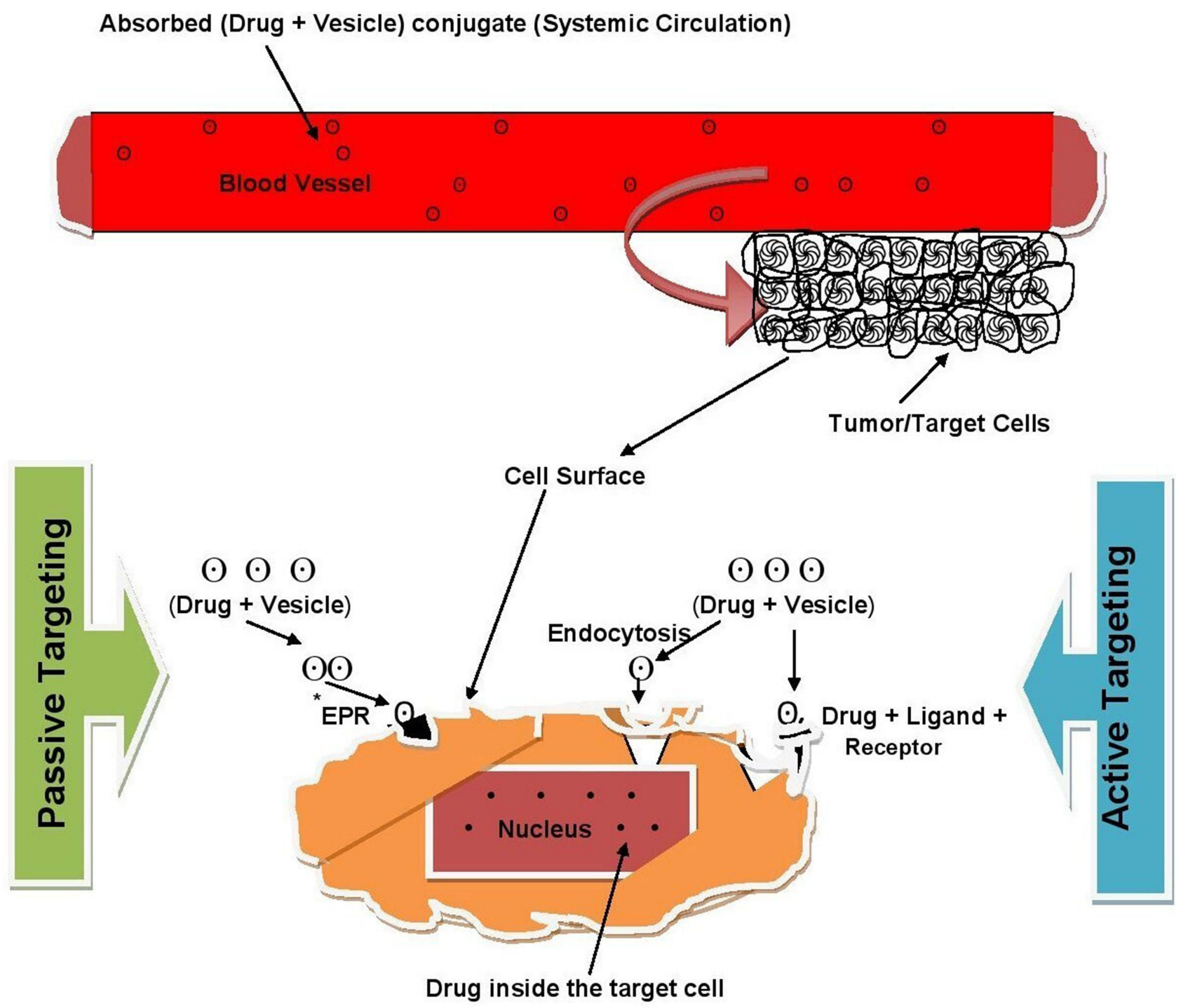

*EPR: Enhanced Permeability \& Retention

Figure 5 Active vs passive targeting.

properties are believed to be suited for passive targeting of anticancer drugs, at relatively smaller dimensions than abnormal blood-vessel gaps, and can easily reach and selectively localize tumors. ${ }^{22}$ However, recent studies have demonstrated that interendothelial gaps on tumors are not responsible for NP transport and accumulation into solid tumors. One study found that active processes take place for up to $97 \%$ of NP transport into tumors through endothelial cells. These investigations suggest the EPR effect in clinical cases in oncology patients has not yet been proven. On the other hand, active transport mechanisms are predicted to be better for uptake of targeted NPs from the bloodstream into the tumor microenvironment than more passive transport mechanisms like the EPR effect. ${ }^{23}$ Though the development of NPs for targeting brings many promising advantages, the efficacy of current nanodrugs is not significantly better than the original drug treatments, especially in cancer chemotherapy. This is because of poor and incomplete nanodrug penetration into tumor tissue, due to the complex features associated with nanodrug size and tumor pathology. Remodeling the microenvironment of tumor tissues, carrier charge inversion, dimensional change, and surface modification are among the demonstrated strategies for 
promoting tumor penetration of nanodrugs. Even with promising penetration-enhancing strategies, effective application of targeting of tumors is far from complete, and cancer chemotherapy still has many difficulties and challenges. As such, DD and targeting systems of a more bioresponsive nature, reduced side effects, and better treatment efficacy are required. ${ }^{24}$

\section{First-, Second-, Third-, and Fourth-Order Targeting}

Drug targeting can further be classified into three (or four) different orders of targeting. In first-order targeting, there is limited distribution of the drug-carrier system to the capillary bed of the target site. Second-order targeting refers to the selective provision of drugs to specific cell types, such as tumor cells. Third-order targeting indicates targeting intracellular sites specifically, and fourth-order targeting is sometimes nominated for drugs targeting macromolecules, such as DNA and proteins. ${ }^{15}$

\section{Inverse, Dual, Double, and Combination Targeting}

If the normal activity of reticuloendothelial system is inhibited by a blank colloidal carrier to minimize its passive drug uptake, the system will be saturated with suppression of its defense mechanisms, an approach known as inverse targeting. Dual targeting is the delivery of carrier molecule with its own therapeutic activity and thus increasing the (synergistic) therapeutic effect of the drug. In double targeting, temporal and spatial methodologies are combined, ie, spatial placement to specific sites and temporal delivery at a controlled rate. Combination targeting is a way of targeted delivery equipped with carriers, polymers, and homing devices of molecular specificity that provide a direct approach to a target. ${ }^{3,20}$

\section{Physical, Chemical, and Biological Targeting}

Physical targeting describes systems that localize agents to target areas because of their size, composition, or other characteristics that are not specifically designed toward a biological receptor. Chemical targeting involves the localization of agents to targeted areas through the use of sitespecific prodrugs. Agents can also be directed to areas through the use of enzymatic or chemical reactions that lead to the targeting of a vehicle or the controlled release or action of the agent. Biological targeting allows localized agents to target areas through the use of antibodies (Abs), peptides, proteins, or other biomolecules that have affinity with receptors, sites, or other biological targets in a specific manner. Gene expression can also be localized to target areas through the use of cells, tissue, or other specific promoters in vector systems. ${ }^{25}$

\section{Local and Systemic Targeting}

Locally targeted systems are noninvasive targeting strategies with the pricipal goal of delivering the drug to the local site for the management of local pathologies. With systemic targeting, delivery of such therapeutic systems occurs through an invasive route, such as intravenous administration of nanotechnological systems. Such systems deliver the drug via systemic circulation after distribution in the body. The major limitations of such systems arise from the adverse effects of the drugs in aspecific tissue. $^{26}$

\section{Location-Based and Disease-Based Targeting}

TDD with specific location-based strategies is a targeted delivery to specific cells, organs, and organelles. Intracellular targeting, gastrointestinal tract (GIT) targeting, brain targeting, and targeting the respiratory tract are some examples of location-based targeting. Intracellular delivery of pharmaceutical agents like proteins, Abs, and drug-loaded nanocarriers ensures that the therapeutic action is specifically introduced to the nucleus or specific organelles. Floating DD is a model for this type of targeting in which antiviral, antifungal, and antibiotic agents are absorbed from very specific regions of the GIT. Various site-specific oral controlled-release systems have been developed to target the stomach/duodenum, small intestine, lymph nodes, and colon. Polymer-based DDSs like dopamine-liposome conjugates show effective brain targeting with reduced degradation during circulation, whereas disease-based targeted delivery is a site-specific therapy targeting tumors and other targetable infectious diseases. Tackling infections using nano-DDSs could provide a practical alternative to antibiotic therapy. Designing nanovaccines to achieve advanced targeting and improved cellular responses is a new prospect. Specific and specialized approaches of targeting some important pathogens for persistence inside the cell are being developed. These include functionalization of NPs with antimicrobial agents. ${ }^{2,17,19,26}$ 


\section{Vehicle/Carrier Systems for Targeted DD}

Drug carriers, sometimes called drug vectors, are the most important entity required for successful transportof the loaded drug for to intended targeted. They transport, retain, and deliver the drug within or at the localion of the target. They are capable of performing such specific functions by slight virtue of structural modification. Currently, DD technology has become refined by the consideration of several factors such as bioavailability, pharmacokinetic processes, and timing for optimal DD. ${ }^{17,27}$ Depending on the type of targeting mechanism, TDDSs require specific carrier systems. TDD carriers/vehicles are purposely engineered vectors capable of retaining the drug inside or on them via encapsulation and/or via bonding with the help of a spacer moiety. These DD vehicles are used for polymeric carriers like micelles, liposomes, lipoprotein-based carriers, and NP-based carriers. ${ }^{28,29}$

Ideally, carriers for TDD must be atoxic, stable, nonimmunogenic, biocompatible, and biodegradable, readily eliminated from the body, and unrecognizable by the host's defense mechanisms. In addition, they should successfully transport the drug to the target site, cross barriers and tumor vasculatures as needed, be of acceptable size and shape (for nanocarriers), and have optimum release properties at the target site, but no or minimum drug leakage before that target site. Carriers should also have reasonably simple, reproductive, and cost-effective preparation processes. ${ }^{28,29}$ The DDS should have optimal target selectivity and specificity, which can be achieved by controlling the biodistribution profiles of the drug and carrier material. The physicochemical and biochemical properties of both parties are used to determine the biodistribution profile. ${ }^{30}$ Similarly, drug release and polymer biodegradation are important factors in developing a successful nanoparticulate system. In sum, solubility, diffusion, and biodegradation of the matrix materials are important for the release process. ${ }^{31}$

\section{Commonly Used Carriers for Targeted Drug Delivery}

There are different types of drug carriers, such as colloidals, polymers, monoclonal Abs, NPs, and cell. The nature of the drug, the target, and the disease state determine the selection of the carrier to be used. Abs, proteins, lipoproteins, hormones, charged molecules, and polysaccharides are used with carriers as targeting moieties. ${ }^{16}$

\section{Colloidal Carrier Systems}

Colloidal DDSs are nanoscaled targeting vesicles of particulate or vesicular dosage form. They include liposomes, niosomes, nanospheres, multiple emulsions, and ceramics. These type of drug vectors sequester, transport, and retain the active drug en route, while they elute or deliver it within or in the vicinity of the target, with the ability to modify the distribution profile. They are commonly categorized as vesicular and microparticulate systems. ${ }^{32}$

\section{Vesicular Carrier Systems}

Novel vesicular DDSs have the objective of delivering the drug at a rate- and site-controlled manner as per treatment needs. Recently, these types of carriers have been emerging with various routes of administration for targeted and controlled DD. ${ }^{33}$ Vesicular DDSs are used to improve the therapeutic index, solubility, stability, and rapid degradation of drug molecules. ${ }^{34}$ Nanosomes are the best-known advance in vesicular carrier systems. They are small, vesicular carrier structures for the delivery of drugs to the target area. They are available in different forms, eg, liposomes, niosomes, transferosomes, and ethosomes. All these vesicular carriers are nanosome generations with modifications of one from the other in their vesicular characteristics and composition during preparation, conditions of preparation and storage, and intended therapeutic applications. ${ }^{35,36}$ These structural and compositional differences are summarized in Table 1.

Liposomes are simple, microscopic, nanoscaled lipoidal vesicles that have a lipid-bilayer structure. These bilayers consist of an aqueous core entirely enclosed by a membrane that is composed of lipid molecules in such a way that both hydrophilic and lipophilic drugs can be successfully entrapped. The bilayer membrane captures lipophilic drugs, whereas hydrophilic drugs will be entrapped in the central aqueous core. ${ }^{37}$ Liposomes are synthesized for use in targeted oral, topical, and pulmonary DD. ${ }^{38}$ Liposomes are composed of many internal and external constituents and layers. Generally, liposome composition includes natural and/or synthetic phospholipids, cholesterol, hydrophilic polymer-conjugated lipids, and water. In most cases, cholesterol is used to improve membrane fluidity and bilayer stability while reducing the permeability of water-soluble molecules through the membrane. Liposomes have a special advantage due to the use of physiological lipids to form the lipid membrane, which decreases the risk of toxicity. ${ }^{39,40}$ They can 
Table I Differences in nanosomal vesicular carriers

\begin{tabular}{|l|l|l|l|}
\hline Nanosome & \multicolumn{1}{|c|}{ Main component } & \multicolumn{1}{c|}{ Uses } & \multicolumn{1}{|c|}{ Special properties } \\
\hline Niosomes & $\begin{array}{l}\text { Cholesterol, charge-inducing substances, } \\
\text { nonionic surfactants }\end{array}$ & Carrier of lipophilic and amphiphilic drugs & $\begin{array}{l}\text { Stable, no need for special } \\
\text { storage or preparation }\end{array}$ \\
\hline Liposomes & Phospholipids dispersed in aqueous solution & $\begin{array}{l}\text { Used in targeted oral, topical, and } \\
\text { pulmonary DD }\end{array}$ & $\begin{array}{l}\text { Unstable, needs special storage } \\
\text { and preparation }\end{array}$ \\
\hline Transferosomes & $\begin{array}{l}\text { Surfactants, a little alcohol, dye, } \\
\text { phosphatadylcholine in buffer solution }\end{array}$ & $\begin{array}{l}\text { Penetrate deeper epidermis layers, used } \\
\text { for transdermal delivery }\end{array}$ & $\begin{array}{l}\text { Ultraflexible and deformable } \\
\text { vesicles }\end{array}$ \\
\hline Ethosomes & $\begin{array}{l}\text { High concentration of alcohol, phospholipid, } \\
\text { water, cholesterol, dye }\end{array}$ & Controlled transdermal delivery & Soft and novel vesicles \\
\hline
\end{tabular}

be formulated in liquid dosage forms (suspension), solid forms (dry powder), or semisolid preparations (gel, cream). They can also be administered parenterally or topically. ${ }^{39}$ After systemic (usually intravenous) administration, liposomes accumulate inside the body simply due to their prolonged circulation and small size, enabling them to extravasate. Topical applications of liposomes are based on the similarity between lipidvesicle bilayers and natural membranes, including the ability of lipid vesicles to alter cell-membrane fluidity and to fuse with them. In the dermatological field, liposomes were initially used because of their moisturizing and restoring action. ${ }^{41}$

Niosomes are nanometric NDDSs in which the medication is encapsulated in vesicles composed of a bilayer of nonionic active surface agents, hence the name. They are promising vehicles for DD, and being nonionic, they are less toxic and improve the therapeutic index of the drug by restricting its action to target cells. Niosomes and liposomes are similar in physicochemical properties, with some differences depending on bilayer composition and preparation methods. While liposomes are composed of phospholipids, niosomes have a major component of surfactants. Niosomes need no special conditions of preparation or storage, since they have very good stability, as opposed to liposomes. This can reduce production costs. ${ }^{42}$ However, their poor skin permeability, breaking of vesicles, leakage of drug, aggregation, and fusion of vesicles limits the suitability of liposomes and niosomes for transdermal delivery. Transferosomes, which are recently introduced carrier systems, overcome these challenges and become capable of effectively delivering low- and high-molecular weight drugs through the transdermal route. ${ }^{43}$
Transferosomes are specially optimized, ultradeformable (ultraflexible) lipid supramolecular aggregates that are able to penetrate mammalian skin intact. They are composed of an inner aqueous compartment and a surrounding lipid bilayer incorporated with edge-activator surfactants like sodium cholate, Span 80, and Tween 80 . They act as penetration enhancers, disrupting the highly organized intercellular lipids from the stratum corneum and facilitating drug penetration in and across the stratum corneum. ${ }^{44}$ Therapeutically, they are used as carriers for proteins and peptides like insulin, bovine serum albumin, and vaccines. They improve site specificity, upgrade overall drug safety, and lower doses of drugs for skin diseases. Because of their good penetration and flexibility, they are used for effective delivery of nonsteroidal anti-inflammatory drugs like ibuprofen and diclofenac. ${ }^{43}$ Ethosomes are other forms of vesicular carriers that are used for delivery of drugs. They have low penetration through biological membrane, mainly skin, bute relatively higher skin penetration than liposomes. They contain phospholipids, alcohol (ethanol and isopropyl alcohol), and water, and are used mainly for transdermal DD. Ethosomes are used as replacements of liposomes, mainly for the transdermal route of DD, and can be used for transdermal delivery of hydrophilic and impermeable drugs. ${ }^{44}$

\section{Microparticulate Systems}

Microparticles are DDSs on the micrometer-millimeter scale. This microencapsulation technology allows protection of the drug from the environment, stabilization of sensitive drug substances, elimination of incompatibilities, and masking unpleasant taste. As such, they play an important role as DDSs, aiming at improved bioavailability of conventional drugs and minimizing side effects. 
Microparticulate systems includes microparticles, NPs, and magnetic microspheres. ${ }^{45}$

\section{Polymeric Carriers for TDDSs}

Polymers are the backbone of pharmaceutical DDSs. They have been found extensive applications in DD because they offer unique properties that have not been attained by any other material. Advances in polymers have led to the development of several NDDSs, with proper consideration of surface and bulk properties contributing to make medical treatment more efficient, effective, and safe. Polymers play an important role in advanced DDSs, as they can used to assist delivery and as excipients, and they allow controlled and targeted drug release. ${ }^{46}$ Micro- and nanospheres fabricated from a biodegradable polymer enable controlled drug release at desired sites. Polymeric nanocarriers, such as poly(D,L-lactide-co-glycolide) have shown promising pharmacokinetics at both the wholebody and cellular levels. ${ }^{47}$ Generally, polymer-based drug nanocarriers can significantly increase the solubility of hydrophobic drugs, reduce their cytotoxicity toward normal tissue, prolong the circulation time of drugs in blood, facilitate the entry of NPs, and improve utilization efficiency. Though natural polymers, such as chitosan and dextran, have been well investigated in the last few decades, research on using synthetic polymers, such as polyesters, polyamides, and polypeptides, has been more prevalent in the field of DD. ${ }^{48}$

Amphiphilic polymers that contain both hydrophilic and hydrophobic blocks have been extensively studied for use in DD as polymeric carriers, such as micelles, nanomicelles, and dendrimers. By controlling the hydrophilic-hydrophobic balance, various nanostructures, such as spherical micelles, cylindrical micelles, and vesicles, can be formed from amphiphilic polymers. Both polymeric micelles and vesicles are the commonest and stablest morphological structures of amphiphiles in water. Polymeric micelles are nanostructures with a hydrophobic core and a hydrophilic shell, where hydrophilic drugs are encapsulated in the core. Meanwhile, polymeric nanovesicles possess bilayer structures with an aqueous interior core, isolating the core from the external medium, where hydrophilic drugs can be encapsulated within the aqueous interior, integrating the hydrophobic molecules within the membrane. Therefore, polymeric vesicles have the capability to deliver hydrophilic and hydrophobic drugs, such as anticancer drugs, genes, and proteins. ${ }^{48}$
Polymeric micelles are composed of a hydrophobic internal core and hydrophilic external surface in which physical entrapment of drug molecules occurs in the hydrophobic core, escaping the need for encapsulationfunctional groups. Loading can be boosted by chemically conjugating the micelles with amphiphilic polymers, which prevents early drug release. The hydrophobic core enhances transportof low or no aqueous solubility, which can improve the therapeutic window of lipophilic drugs. This further prevents the formation of embolisms of intravenously administered hydrophobic drugs. Micelles are also characterized by decreased chances of rapid drug clearance and prolonged circulation in vivo that encourages drug accumulation at tumor cells. These characteristics make polymeric micelles an important newgeneration nanomedicine with advanced diagnostic and therapeutic clinical applications. ${ }^{49,50}$

Dendrimers are the other types of polymeric carriers for drug targeting. They are monodispersed macromolecules with well-articulated and structurally multibranched globular units. Dendrimers consist of three main parts with particular functions: focal point, interior branching units, and exterior surfaces with functional groups (their function is described in Table 2). Both polar and apolar drugs are trapped into dendrimers by electrostatic interaction and hydrophobic fragmentation, respectively. Drug molecules can either be attached to dendrimers' interior cavity noncovalently with physical interaction or covalently linked to the exterior groups. Gene plasmids and nucleic acids are examples that can be linked through electrostatic interaction. The covalent linkage offers a more stable formulation for DD. Drug release is determined by the nature of the linkage. ${ }^{49}$ For a successful dendrimer DDS, the critical nanoscale design parameters - particle size, shape, surface, flexibility/rigidity, architecture, and elemental composition - should be well considered. $^{51}$

\section{Monoclonal Antibodies and Fragments}

Monoclonal Abs (mAbs) are getting attention as therapeutic agents in targeting for the treatment of various chronic conditions, such as cancer and infectious diseases. They can be conjugated with chemotherapeutic drugs, radioisotopes, bacterial toxins, cytokines, and enzymes for targeting of tumors in order to potentiate their cytotoxic effects. Nowadays, human mAbs are being formulated as antitumor agents. For example, adalimumab is the first human $\mathrm{mAb}$ officially approved for clinical use. ${ }^{3}$ 
Table 2 Structural components of dendrimers

\begin{tabular}{|l|l|}
\hline Structural Components & \multicolumn{1}{|c|}{ Description/Function } \\
\hline Focal point (core) & The center of the dendrimer can be a small molecule, nanoparticle, or polymeric material. \\
\hline Free (void) spaces & $\begin{array}{l}\text { These are empty spaces between the core and interior branchings to be used as room for drug encapsulation or } \\
\text { carrying. }\end{array}$ \\
\hline Interior branching & $\begin{array}{l}\text { Multibranched globular units with internal functional groups that have a covalent framework that connect the } \\
\text { dendrimer core with the outer-surface groups. }\end{array}$ \\
\hline Exterior groups & $\begin{array}{l}\text { These are the outer hydrophilic or hydrophobic surface groups that construct the cover of the dendrimer-drug } \\
\text { complex. }\end{array}$ \\
\hline Dendrimer-drug linkage & Covalent or noncovalent bond between the dendrimer and the drug. \\
\hline
\end{tabular}

\section{Recent Advances, Challenges, and Future Perspectives}

In recent years, microsponges, solid-lipid NPs, and nanostructured lipid carriers have been used and further investigated as carrier systems/vesicles for DDSs. Microsponges are synthetic, biologically porous, inert polymers that can carry up to their own weight in drugs. They have the ability to protect the drug from the external environment and to provide controlled release. Nanotechnology has been implemented in several fields of nanomedicine, such as drug/gene delivery, imaging, and diagnostics. $\mathrm{Ab}$ - drug conjugates or immunoconjugates are being investigated as alternative recombinant Abs by covalently binding through a linker to a drug to target potent drugs to specific sites using the specificity of mAbs, thus avoiding nontargeted-organ toxicity. There are also other advances, such as micro- and nanoemulsions, nanocapsules, smart capsules, cyclodextrins, microspheres, nanotubes, nanoshells, quantum dots, hydrogels, metal and magnetic NPs, and natural and synthetic polymeric NPsthat are being investigated for local and systemic targeting. ${ }^{2,52}$

Though there have been promising recent advances, there are also associated challenges in their application. The following sections outline some of the critical challenges.

\section{Challenges Specific to Receptors, Ligands, and Carriers}

Difficulties with identification of receptors, variable expression characteristics, receptor accessibility in terms of reachability and availability, and shedding of receptors are among the challenges specific to receptors. Ligandspecific challenges include appropriate selection of a ligand, developing conjugation strategies of targeting ligands with drug/carrier, and release characterization of drug from ligands (selection of a linker). Carrier-based challenges consist of carrier selection and physicochemical and pharmacokinetic characterization of carriers. ${ }^{53}$

\section{Misconceptions}

There are uncomfortable facts concerning TDD that are overlooked and misconceived. First, targeting is not precise, but implies simply random distribution. Second, the theory of receptor overexpression has not yet fully correlated with targeted delivery. Third, there is improved delivery from the EPR effect, but not as exact as with targeted delivery. Fourth, there may be drug release before the target site, and reaching the tumor tissue does not necessarily mean achieving improved delivery. ${ }^{5}$

\section{Complex Manufacturing Processes}

There is a need for additional steps in chemical synthesis and purification for targeted drug formulation. There are also associated challenges like more quality control and regulatory steps, increased cost, and longer time lines. Scalability, sensitivity, biocompatibility, and toxicity are all associated design challenges for nanocarriers. ${ }^{6,54}$

\section{Tumor Heterogeneity}

Additional complexity exists in the immense heterogeneity within and between tumors. There is also the existence of tumor- and metastasis-associated stroma, eg, tumorassociated macrophages and fibroblasts. ${ }^{54}$

\section{Barely Predictable Practical Outcomes}

There is still substantial debate on the practical outcome of drug-targeting strategies. Lack of clinically translatable models and completely specific targets, along with 
selection of targets with spatial and temporal expression well aligned to interventional requirements, make the success of these approaches hardly predictable. ${ }^{55}$

\section{Barriers in Clinical Translation}

Unproven EPR effect in clinical cases in human oncology, lower-than-expected NP accumulation within tumors with active targeting mechanisms, and factors that should be considered, modified, and controlled during the preparation and delivery of nanomedicines have posed a significant barrier to clinical translation of nanomedicines into human medicine. ${ }^{23}$

NP-based targeting depends on certain physicochemical factors, such as particle size, surface charge, surface modification, and hydrophobicity. There is still limited knowledge on NP toxicity and still many problems related to selective binding and targeted delivery that need to be overcome. Consideration of these problems now and with future NP advances may lead to a new, more prosperous paradigm of therapeutics and research. ${ }^{56}$

There are growing and interesting future perspectives on responding to these challenges and extracting maximum benefit from targeted delivery. Some are briefly stated in the following sections.

\section{Advances in Clinical Extrapolation}

Bursting clinical extrapolation of TDDSs is yet to be proficient. This can be attained only through advanced strategies and reproducible methods of carrier preparations, as well as extensive and elaborative preclinical evaluations. ${ }^{53}$

\section{Cell-Specific Delivery}

Receptor-targeted delivery has a very positive future in areas of identifying newer targets for therapeutics, advanced biological products, and development of various NDDSs for cell-specific delivery. ${ }^{53}$

\section{Theranostic Strategy}

The future of nanomedicine will combine diagnosis and targeted therapy into a single, centralized system of treatment. This novel theranostic strategy brings the potential of a highly selective, effective, and relatively sensitive treatment of cancer and other chronic diseases which further results in personalized chemotherapy with improved patient outcomes. ${ }^{10}$

\section{Advanced Models, Good Laboratory Practice, and Standardization}

The development of targeting strategies should be subjected to continuous evaluation in the light of advances in the understanding of numerous processes occurring after administration. ${ }^{16}$ Developing better and more predictive preclinical animal models, adoption of good laboratory practice and standardization guidelines, better understanding of tumor biology, and identification of actual biomarkers will intensify the accomplishment rate of clinical translation. ${ }^{54}$

\section{Precision Medicine (Engineering Precision NPs)}

Precision medicine is an optimized drug design and DS for individual patients that has changed the setting of cancer chemotherapy remarkably. In precision medicine, a drug's pharmacological and pharmacokinetic parameters are modulated without compromising the anticipated effect at the molecular targets. Precisely, a drug's absorption characteristics, its behavior upon exposure to target and nontarget tissue, and its administration in synergistic drug combinations can be modified. ${ }^{57}$ Interpersonal and interdisease differences in biological barriers against effective DD can be addressed by lipid- based, polymeric and inorganic NPs that are engineered and optimized in increasingly specified ways. These optimized and engineered NPs are entering the era of precision medicine to overcome the heterogeneous biological barriers found across patient populations and diseases. Immunoactivation or -suppression, genome engineering, and intracellular targeting are some of the promising applications of NPs in precision medicine. ${ }^{58}$

\section{From Nanoparticles to Nanorobots}

Pharmacytes, nanorobotic DDSs, are self-powered, computer-controlled systems capable of digitally precise transport, timing, and targeted delivery within the human body. They need not always be endocytosed: nanorobots may use transmembrane mechanical nanoinjectors to avoid having to enter a target cell. The design, fabrication, and therapeutic deployment of pharmacytes will be a promising advance with the application of nanomedicine in the future engineering discipline of medical nanorobotics. ${ }^{59}$

\section{Conclusion}

Nanomedicine is the advanced version of Paul Ehrlich's magic bullet concept. A large variety of NPs can be used to prepare targeted-delivery nanomedicines. TDD is advancing as one of 
the brightest prospects within the medical sciences for the diagnosis and treatment of lethal diseases. Drugs for targeting can easily be attached to conjugated polymers, polymeric micelles, liposomes, dendrimers, and polymeric NPs, due to their characteristic structural features. Many problems related to drug-targeting strategies for clinical application have been identified, analyzed, and solved, especially in the treatment of cancer. Combining expertise with technological developments and interdisciplinary research might help to introduce safer way nanomedicine.

\section{Abbreviations}

DD, drug delivery; DDSs, drug delivery systems; EPR, enhanced permeability and retention; GIT, gastrointestinal tract; NDDSs, novel DDSs; NPs, nanoparticles; TDDSs, targeted DDSs.

\section{Acknowledgments}

Addis Ababa University, Bahir Dar University, and the Ethiopian FDA are thankfully acknowledged for giving us Internet access.

\section{Author Contributions}

All authors contributed to data analysis, drafting or revising the article, have agreed on the journal to which the article will be submitted, gave final approval to the version to be published, and agree to be accountable for all aspects of the work.

\section{Funding}

No funding was provided.

\section{Disclosure}

The authors report no conflicts of interest in this work.

\section{References}

1. Zishan M, Zeeshan A, Faisal S, et al. Vesicular drug delivery system used for liver diseases. World J Pharm Sci. 2017;5(4):28-35.

2. Thakur A, Roy A, Chatterjee S, Chakraborty P, Bhattacharya K, Mahata PP. Recent trends in targeted drug delivery. SMGroup. 2015.

3. Kumar A, Nautiyal U, Kaur C, Goel V, Piarchand N. Targeted drug delivery system: current and novel approach. Int $J$ Pharm Med Res. 2017;5(2):448-454.

4. Akhtar M, Jamshaid M, Zaman M, Mirza AZ. Bilayer tablets: a developing novel drug delivery syste. J Drug Deliv Sci Technol. 2020;60:102079. doi:10.1016/j.jddst.2020.102079

5. Kwon IK, Lee SC, Han B, Park K. Analysis on the current status of targeted drug delivery to tumors. $J$ Control Release. 2012;164:108-114. doi:10.1016/j.jconrel.2012.07.010

6. Yoo J, Park C, Yi G, Lee D, Koo H. Active targeting strategies using biological ligands for nanoparticle drug delivery systems. Cancers. 2019;11:640. doi:10.3390/cancers11050640
7. Ali Y, Alqudah A, Ahmad S, Hamid SA, Farooq U. Macromolecules as targeted drugs delivery vehicles: an overview. Des Monomers Polym. 2015;22:1,91-97.

8. Mishra N, Pant P, Porwal A, Jaiswal J, Samad MA, Tiwari S. Targeted drug delivery: a review. Am J PharmTech Res. 2016;6(1).

9. Fahmy TM, Fong PM, Goyal A, Saltzman WM. Targeted for drug delivery. Mater Today. 2005;8:18-26. doi:10.1016/S1369-7021(05) 71033-6

10. Benyettou F, Motte L. Nanomedicine: towards the "magic bullet" science. J Bioanal Biomed. 2016;8:2.

11. Valent P, Groner B, Schumacher U. Paul Ehrlich (1854-1915) and his contributions to the foundation and birth of translational medicine. J Innate Immun. 2016;8:111-120. doi:10.1159/000443526

12. Gradmann C. Magic bullets and moving targets. Dynamis. 2011;31 (2):305-321. doi:10.4321/S0211-95362011000200003

13. Barz M. Complexity and simplification in the development of nanomedicines. Nanomedicine (Lond). 2015;10(20):3093-3097. doi:10.2217/nnm.15.146

14. Bae YH, Park K. Targeted drug delivery to tumors: myths, reality and possibility. J Control Release. 2011;153:198-205. doi:10.1016/j. jconrel.2011.06.001

15. Rani K, Paliwal SA. Review on targeted drug delivery: its entire focus on advanced therapeutics and diagnostics. Sch J App Med Sci. 2014;2(1C):328-331.

16. Gujral S, Khatri S. A review on basic concept of drug targeting and drug carrier system. IJAPBC. 2013;2(1).

17. Mahajan HS, Patil SB, Gattani SG, Kuchekar BS. Targeted drug delivery systems. Pharma Times. 2007;39(2).

18. Vyas SP, Khar RK. Targeted \& Controlled Drug Delivery: Novel Carrier Systems. New Delhi, India: CBS Publishers \& Distributors; 2004.

19. Manish G, Vimukta S. Targeted drug delivery system: a review. Res $J$ Chem Sci. 2011;1(2):135-138.

20. Bhargav E, Madhuri N, Ramesh K, Manne A, Ravi V. Targeted drug delivery - a review. World J Pharm Pharm Sci. 2013;3(1):150-169.

21. Erkoc P, Cinay GE, Kizilel S. Targeted drug delivery: overcoming barriers through the design of novel delivery vehicles. SMGroup. 2015.

22. Jin K, Lu Z, Chen J, et al. Recent trends in nanocarrier-based targeted chemotherapy: selective delivery of anticancer drugs for effective lung, colon, cervical, and breast cancer treatment. J Nanomater. $2020 ; 14$.

23. Sindhwani S, Syed AM, Ngai J, et al. The entry of nanoparticles into solid tumours. Nat Mater. 2020;19(5):566-575. doi:10.1038/s41563019-0566-2

24. He B, Sui X, Yu B, Wang S, Shen Y, Cong H. Recent advances in drug delivery systems for enhancing drug penetration into tumors. Drug Deliv. 2020;27(1):1474-1490. doi:10.1080/ 10717544.2020.1831106

25. Mills JK, Needham D. Targeted drug delivery. Exp Opin Ther Patents. 1999;9(11):1499-1513. doi:10.1517/13543776.9.11.1499

26. Devarajan PV, Jain S, Editors. Targeted Drug Delivery: Concepts and Design. New York Dordrecht London: Springer Cham Heidelberg; 2015.

27. Bertrand N, Leroux J. The journey of a drug-carrier in the body: an anatomophysiological perspective. $J$ Control Release. 2012;161:152-163. doi:10.1016/j.jconrel.2011.09.098

28. Kivilcım O, Hakan E, Sema Ç. Novel advances in targeted drug delivery. J Drug Target. 2017. doi:10.1080/1061186X.2017.1401076

29. Scott R, Crabbe D, Krynska B, Ansari R, Kiani M. Aiming for the heart: targeted delivery of drugs to diseased cardiac tissue. Expert Opin Drug Deliv. 2008;5(4):459-470. doi:10.1517/17425247.5.4.459

30. Xiaojiao Y, Ian T, Muqing R, Kayla D, Xing G, Chen Z. Design of nanoparticle-based carriers for targeted drug delivery. J Nanomater. 2016;2-12.

31. Idayu IM, Suguna S, Nurul A. Designing polymeric nanoparticles for targeted drug delivery system. Nanomedicine. 2015;288-311. 
32. Bhardwaj A, Kumar L. Colloidal drug delivery systems: a future prospective for treatment of tuberculosis. Am J PharmTech Res. 2011;1(3):102-123.

33. Biju S, Talegaonkar S, Mishra P, Khar K. Vesicular systems: an overview. Indian J Pharm Sci. 2006;68(2):141-153. doi:10.4103/ 0250-474X.25707

34. Sunil K, Vipin S, Nancy M, Suman B, Vikas J. Vesicular drug delivery systems: a novel approach for drug targeting. Int $J$ Drug Deliv. 2013;121-130.

35. Arora S, Lamba HS, Tiwari R. Dermal delivery of drugs using different vesicular carriers. Asian $J$ Pharm. 2012;6:237. doi:10.4103/0973-8398.107558

36. Priya K, Kumar V, Damini VK, et al. Somes: a review on composition, formulation methods and evaluations of different types of "somes" drug delivery system. Int J App Pharm. 2020;12(6):7-18.

37. Anwekar H, Patel S, Singhai AK. Liposome-as drug carrier. Int J Pharm Life Sci. 2011;2(7):945-951.

38. Abu-Dahab R, Schafer UF, Lehr C. Lectin-functionalized liposomes for pulmonary drug delivery: effect nebulization on stability and bioadhesion. Eur J Pharm Sci. 2001;14:37-46. doi:10.1016/S09280987(01)00147-6

39. Laouini A, Jaafar-Maalej C, Limayem FH. Preparation, characterization and applications of liposomes: state of the art. J Colloid Sci Biotechnol. 2012;149-166.

40. Vemuri S, Rhodes C. Preparation and characterization of liposomes as therapeutic delivery systems: a review. Pharm Acta Helv. 1995;70 (2):95-111. doi:10.1016/0031-6865(95)00010-7

41. Jain RK. Delivery of molecular and cellular medicine to solid tumors. J Colloid Sci Biotechnol. 2001;2-8.

42. Shukla A. Niosomes: an approach towards targeted drug delivery system. Int J Pharm Pharm Sci. 2016;6(6):40-46.

43. Elsayed MM, Abdallah OY, Naggar VF, Khalafallah NM. Lipid vesicles for skin delivery of drugs: reviewing three decades of research. Int J Pharm. 2007;1-16.

44. Seema M, Jadhav P, Manisha K, Vilasrao K. Novel vesicular system: an overview. J Appl Pharm Sci. 2012;2(1):193-202.

45. Gattani YS. Floating Multiparticulate drug delivery systems: an overview. Int J Pharma Bio Sci. 2010;6(2):35-40.

46. Ravinder K, Sukhvir K. Role of polymers in drug delivery. J Drug Deliv Ther. 2014;4(3):32-36.
47. Stevanovi M, Uskokovi D. Poly(Lactide- co- glycolide) based micro and nanoparticles for controlled drug delivery of vitamins. Curr Nanosci. 2009;5(1):1-15. doi:10.2174/157341309787314566

48. Haijiao L, Jingkang W, Ting W, Jian Z, Ying B, Hongxun H. Recent progress on nanostructures for drug delivery applications. J Nanomater. 2016;2-9.

49. Yousaf A, Ali A, Sadiq A, Shafida A, Umar F. Macromolecules as targeted drugs delivery vehicles: an overview. Des Monomers Polym. 2019;22(1):91-97. doi:10.1080/15685551.2019.1591681

50. Batrakova EV, Bronich TK, Vetro JA, Kabanov AV. Polymer micelles as drug carriers. In: Torchilin VP, editor. NPs as Drug Carriers. London: Imperial College Press; 2006:57-93.

51. Rav S, Maina SS, Malviya R, Kushwah MG, Khan MG, Yezdani U. Novel approach of targeted drug delivery system and its application. ARC J Public Health Commun Med. 2019;4(4):1-4.

52. Martinho N, Damgé C, Reis CP. Recent advances in drug delivery systems. J Biomater Nanobiotechnol. 2011;2:510-526. doi:10.4236/ jbnb.2011.225062

53. Vhora I, Patil S, Bhatt P, Gandhi R, Baradia D, Misra A. Receptortargeted drug delivery: current perspective and challenges. Ther Deliv. 2014;5(9):1007-1024. doi:10.4155/tde.14.63

54. Rosenblum D, Joshi N, Tao W, Karp JM, Peer D. Progress and challenges towards targeted delivery of cancer therapeutics. Nat Commun. 2018;9:1410. doi:10.1038/s41467-018-03705-y

55. Freitas RA. Pharmacytes: an ideal vehicle for targeted drug delivery. $J$ Nanosci Nanotechnol. 2006;6:2769-2775. doi:10.1166/ jnn.2006.413

56. Mirza AZ, Siddiqui FA. Nanomedicine and drug delivery: a mini review. Int Nano Lett. 2014;4(1):94. doi:10.1007/s40089-014-0094-7

57. Manzari MT, Shamay Y, Kiguchi H, Rosen N, Scaltriti M, Heller DA. Targeted drug delivery strategies for precision medicines. Nat Rev Mater. 2021;6:351-370.

58. Mitchell MJ, Billingsley MM, Haley RM, Wechsler ME, Peppas NA, Langer R. Engineering precision nanoparticles for drug delivery. Nat Rev Drug Discov. 2021;20:101-124.

59. Muro S. Targeted drug delivery systems: achievements and challenges. J Nanomedic Nanotechnol. 2012;3:1.
Journal of Multidisciplinary Healthcare

\section{Publish your work in this journal}

The Journal of Multidisciplinary Healthcare is an international, peerreviewed open-access journal that aims to represent and publish research in healthcare areas delivered by practitioners of different disciplines. This includes studies and reviews conducted by multidisciplinary teams as well as research which evaluates the results or conduct of such teams or healthcare processes in general. The journal covers a very wide range of areas and welcomes submissions from practitioners at all levels, from all over the world. The manuscript management system is completely online and includes a very quick and fair peer-review system. Visit http://www.dovepress.com/testimonials. php to read real quotes from published authors. 